\title{
Image Encryption Method based on Hill Matrix and Dynamic DNA Encoding
}

\author{
Xuncai Zhang, Zheng Zhou, Yishan Liu, Guangzhao Cui, Ying Niu*, and Yanfeng Wang \\ School of Electrical and Information Engineering, Zhengzhou University of Light Industry, Zhengzhou, 450002, China
}

\begin{abstract}
Based on Hill matrix and dynamic DNA encoding, an image encryption method is proposed. First, this method combines an elliptic curve with a hyper-chaos sequence to fabricate the Hill cipher matrix, and the plaintext image is permuted and encrypted. Second, the dynamic DNA encoding technique is applied to diffuse the gray values of this plaintext image. Finally, hyper-chaos sequences are employed to complete the confusion and diffusion of the image. The experimental results show that the presented method can not only defend against brute-force attacks and statistical attacks but also resist plaintext attacks; in addition, the method has the merits of fast encryption speed, good encryption effects, and easy implementation.
\end{abstract}

Keywords: encryption method; DNA coding; hHill matrix; hyper-chaos system; elliptic curve

(Submitted on May 6, 2018; Revised on June 19, 2018; Accepted on July 21, 2018)

(C) 2018 Totem Publisher, Inc. All rights reserved.

\section{Introduction}

Digital images are a common information communication method because of their features, including intuitiveness, ease of recognition, vividness, high redundancy, and ability to convey a large amount of data. Digital images are vulnerable to attacks from unauthorized network transmissions, so encryption technology is utilized to guarantee the reliability of information transmissions. Some conventional encryption algorithms such as RC4 and Blowfish do not take into account the strong correlation between pixels in the digital image itself. Therefore, these image encryption methods have many problems such as low encryption intensity or excessively long encryption time. Therefore, finding new image encryption methods is currently a popular research topic.

A hyper-chaos system has a multitude of properties, such as unpredictable orbit and sensitivity to initial state and control parameters. Hyper-chaotic sequences have a series of advantages, such as a long prediction period, good randomness, large key space, high-security performance, and difficulty with cracking [1-2]. DNA molecules have advantages of ultra-large parallelism and ultra-high storage space. The combination of hyper-chaotic sequences and DNA encoding can solve the shortcomings of the singleness of DNA encoding and make up for the low sensitivity of the hyperchaos sequence to the key. In literature [3], an encryption scheme based on hyper-chaotic and dynamic DNA coding is introduced that adds DNA coding rules. However, this algorithm has only one kind of DNA operation in the encryption process and has a single calculation rule. Literature [4] demonstrates that the hyper-chaos encryption algorithm using fixed coding and single-operation rules is likely to crack by a plaintext attack. Other research [5-6] put forward an encryption method based on DNA encoding and multiple chaos maps, where a hyper-chaos system is introduced to confuse the pixel values, and the DNA encoding rules are exploited to conduct the pseudo-DNA manipulation; in addition, the ciphered image is finally gained by DNA decoding. Although DNA encoding and hyper-chaotic Chen system provide good encryption, they do not fully show forward-secrecy because of their symmetric properties. To address these problems, some scholars have proposed using public key cryptography to make up for the lack of effective key management for symmetric cryptography.

\footnotetext{
* Corresponding author.

E-mail address: niuying@zzuli.edu.cn
} 
At present, there exist two kinds of encryption technologies: symmetric encryption and asymmetric encryption. Asymmetric encryption is suitable for multiple users to communicate securely in public networks. Applications of public key cryptography mainly include RSA cryptosystem, ElGamal cryptosystem, and elliptic curve cryptosystem. Because the elliptic curve cryptosystem (ECC) [7] is under the same security intensity, it has the advantages of a short key, flexible parameter selection, high-security, and fast encryption, and it is widely used in security authentication, digital signature, and data security transmission systems [8]. One study proposed an algorithm to embed image data into an elliptical curve with the features of an image. After processing, the image can still be restored according to the elliptic curve point group operation. Another study [9] proposed to encrypt the pixel values of digital images; the disadvantage is that the encryption and decryption processes take more time. A third study [10] proposed a block-image method based on ECC that divides the scrambled matrix into the image elements and then performs the elliptic encryption transformation; however, the pixel quality of block pixels in the process of sending is higher, and the encryption and decryption processes are complicated. Another analysis [11] used the characteristics of the image itself and combined the encryption algorithm of the elliptic curve to apply the elliptic curve public key cryptography to digital image encryption. However, the algorithm will take a long time to encrypt and decrypt. In an article [12], DNA computing and ECC were used to encrypt the RGB images. However, the sensitivity of the algorithm to the parameters was not high. Bibhudendra et al. proposed to construct a self-inverse matrix [13-14] to encrypt the information to overcome the weakness of the inverses of the encrypt matrix. Since the construction of the inverse matrix adopts pseudo-random numbers and the elements are strongly correlated, it is easy to crack. In literature [15], chaotic sequences are used to construct the encryption matrix, but the encryption method is relatively simple. Other research [16] suggests constructing the Hill encryption matrix by an elliptic curve, but the algorithm is too complex.

As described in this method, the technology of a hyper-chaos system and an elliptic curve is utilized to construct the encryption matrix to permute and encrypt the plaintext, avoiding the strong correlation between elements and the complexity and difficulty of elliptic curve encryption. Then, the encoding rules are randomly selected according to the different position of the pixels, and the pixels are encoded by DNA. Finally, the hyper-chaos sequence is used to scramble the plaintext. The merit of the method is that it raises the sensitivity of the key, effectively resisting known and chosen plaintext attacks and having a preferable capacity to anti-brute force attacks and defend against differential attacks.

\section{Hyper-Chaotic System and DNA Encoding Rules}

\subsection{Hyper-Chaos Lorenz System}

In 1963, Lorenz obtained a series of basic features of chaotic motion by observing a large number of atmospheric phenomena and conducting numerical experiments and theoretical analysis. The first singular attractor-Lorenz attractor was proposed. Lorenz simulated a fourth-order differential equation by computer. The weather model described found that under certain conditions, the same system can exhibit acyclic irregular behaviour. Lorenz revealed the basic characteristics of a series of chaotic motions, which became the cornerstone and starting point of future generations of chaos theory. It is of great significance in secret communication. The mathematical expression of the hyper-chaos Lorenz system [17] is described as follows:

$$
\left\{\begin{array}{l}
\dot{x}=\alpha(y-x)+u \\
\dot{y}=\gamma x-y-x z \\
\dot{z}=x y-\beta z \\
\dot{w}=-y z+\delta u
\end{array}\right.
$$

Where $\alpha, \beta, \gamma$, and $\delta$ are system parameters and $x, y, z$, and $u$ are state variables, When $\alpha=10, \beta=8 / 3, \gamma=28$ and $\delta=-1$, the system is in hyper-chaos state, and four chaotic sequences are generated. The four Lyapunov exponents of the formula (1) are: $\lambda_{1}=-15.1752, \lambda_{2}=0, \lambda_{3}=0.1586, \lambda_{4}=0.3381$. Because the hyper-chaotic system has two positive Lyapunov exponents, the computational speed of the hyper-chaos system is faster than that of the normal chaotic system. Therefore, it is more secure to design cryptographic algorithms.

\subsection{DNA Encoding and Sequence Operations}

\subsubsection{Dynamic DNA Encoding}

A DNA sequence is made up of four nitrogenous bases [18], that is to say, adenine (A), cytosine (C), guanine (G) and thymine (T) [19]. The well-known Watson-Crick base pairing rule states that A complements with $\mathrm{T}$, and $\mathrm{G}$ complements with $C$ [20]. When 0 and 1 are two mutually complementary relationships, 00 and 11,01 and 10 are two mutually 
complementary relationships, respectively. The pixel value is expressed by an 8-bit binary digit. Under the circumstance, there are eight coding rules that meet the base pairing rules, as shown in Table 1. The pixel value of a grayscale image is between 0 and 255, so it is described as an 8-bit binary digit or a 4-bit DNA encoding. For example, the decimal number 133 as an 8-bit binary number is 10000101, with rule 3 encoding as ACTT and rule 8 decoding as 11100000 . The information can be encrypted only by the difference of encoding and decoding methods.

Table 1.8 Encoding rules

Table 1.8 Encoding rules
\begin{tabular}{|c|c|cc|ccc|cc|}
\hline Rule & 1 & 2 & 3 & 4 & 5 & 6 & 7 & 8 \\
\hline 00 & A & C & C & A & T & G & G & T \\
\hline 01 & G & A & T & C & C & A & T & G \\
\hline 11 & T & G & G & T & A & C & C & A \\
\hline 10 & C & T & A & G & G & T & A & C \\
\hline
\end{tabular}

For the given image $I(m, n)$, the DNA encoding rule is chosen as shown in formulas (2) or (3):

$$
\begin{gathered}
r_{i, j}=(\operatorname{round}(\bmod ((i+j), 8))+1) \\
r_{i, j}^{\prime}=(\operatorname{round}(\bmod (i \times j, 8))+1)
\end{gathered}
$$

Where $i$ and $j$ represent the row and column of the pixel, $i \in\{1,2, \cdots, m\}$, and $j \in\{1,2, \cdots, n\}, P(i, j)$ in the matrix, respectively. The encoding rules are selected according to the pixel position, which increases the diversity of coding rules.

\subsubsection{DNA Encoding Operations}

The DNA subtraction and addition operation rules are similar to a conventional algebraic computation. The definition of rules between bases is defined as shown in Table 2 .

Table 2. DNA subtraction and addition operation rules

\begin{tabular}{|c|c|c|c|c|c|c|c|c|c|}
\hline- & A & G & C & T & + & A & G & C & T \\
\hline A & A & T & C & G & A & A & G & C & T \\
\hline G & G & A & T & C & G & G & C & T & A \\
\hline C & C & G & A & T & C & C & T & A & G \\
\hline T & T & C & G & A & T & T & A & G & C \\
\hline
\end{tabular}

\section{Scheme Design}

The Hill encryption matrix is constructed by combining a hyper-chaos system and elliptic curve, and the image is replaced and encrypted. Next, the dynamic DNA encoding and hyper-chaotic sequence operations are utilized to realize the confusion and scrambling of the image.

\subsection{The Key Generation of Hyper-Chaos System}

The SHA-3 function is also called the hash function [21], which is the most basic components in modern cryptosystem, and the input data is variable length and the output is fixed length. Messages can be generated with hash functions to generate a message digest that is appended to a message or stored with a message to prevent the message from being tampered with in transit and storage. The hash value of two images is completely different even if only one bit is different. Using this feature, a new sequence is generated with a set key and a summary of the image, the appropriate hash function is selected to generate the hash value of the new sequence, and then the security of encryption is improved by handling the initial values and system parameters of the modified chaotic system accordingly. By combining the plaintext with the key, the computational complexity is $2^{512}$, so the encryption method can effectively resist known plaintext and brute force attacks.

After the plaintext is operated with SHA-3 (256), a block of 256-bit values are obtained, and the hash value is converted to binary as the key $a$. The key $a$ is further processed with Hamming distance to produce the key of the hyper-chaos Lorenz system. The keys of this generation have the advantages of randomness and periodicity.

The Hamming distance represents the number of different characters of two equal-length strings at their corresponding position [22]. From another perspective, it measures the minimum character of substitutions required to convert the string $x$ to a $y$ by replacing the character. Assuming: $x=\left\{x_{1}, x_{2}, \cdots, x_{i}\right\}, y=\left\{y_{1}, y_{2}, \cdots, y_{i}\right\}$, the Hamming distance formula for 
calculating these two sequences is as follows:

$$
\left\{\begin{array}{l}
H(x, y)=\sum_{i=1}^{n} h\left(x_{i}, y_{i}\right) \\
h\left(x_{i}, y_{i}\right)= \begin{cases}0, & x_{i}=y_{i} \\
1, & x_{i} \neq y_{i}\end{cases}
\end{array}\right.
$$

The key $a$ is divided by bytes and can be divided into 32 bytes, expressed as follows: $a_{1}, a_{2}, \cdots, a_{32}$. For example, $b_{1}=11011101, b_{2}=11001001$, so the Hamming distance $H\left(b_{1}, b_{2}\right)$ of the two sequences is 2 . If $C_{1}=a_{1} \oplus a_{2} \oplus a_{3} \oplus a_{4} \oplus a_{5} \oplus a_{6}$ $\oplus a_{7} \oplus a_{8}, C_{2}=a_{9} \oplus a_{10} \oplus a_{11} \oplus a_{12} \oplus a_{13} \oplus a_{14} \oplus a_{15} \oplus a_{16}, C_{3}=a_{17} \oplus a_{18} \oplus a_{19} \oplus a_{20} \oplus a_{21} \oplus a_{22} \oplus a_{23} \oplus a_{24}, C_{4}=a_{25} \oplus a_{26} \oplus a_{27} \oplus a_{28}$ $\oplus a_{29} \oplus a_{30} \oplus a_{31} \oplus a_{32}$. The key of the hyper-chaos Lorenz system is calculated by the formula (5), where $x_{1}, y_{1}, z_{1}$, and $u_{1}$ are given values.

$$
\left\{\begin{array}{l}
x_{0}=x_{1}+H\left(C_{1}, C_{2}\right)+C_{1} / 4 \\
y_{0}=y_{1}+H\left(C_{2}, C_{3}\right)+C_{2} / 4 \\
z_{0}=z_{1}+H\left(C_{3}, C_{4}\right)+C_{3} / 4 \\
u_{0}=u_{1}+H\left(C_{4}, C_{1}\right)+C_{4} / 4
\end{array}\right.
$$

\subsection{The Construction of $M$ Sequence}

Given the grayscale image $I(m, n)$, set $L=[m \times n / 8]$, the chaotic sequence generated by the hyper-chaos Lorenz system is applied to construct $L / 16$ new sequences $-M$ sequence. The specific construction process is shown below:

Step 1 Fabricate $L / 16$ empty sequence $M_{1}, M_{2}, \cdots, M_{L / 16}$, and each sequence length is 256 .

Step 2 The interval $[0,256)$ is separated into 256 sub-intervals $T_{j}=[j, j+1)$, where $j=0,1, \cdots, 255$.

Step 3 According to the keys $x_{0}, y_{0}, z_{0}$, and $u_{0}$, the hyper-chaos Lorenz system is iterated $\mathrm{s}(\mathrm{s}>\mathrm{m} \times \mathrm{n})$ times by using formula (1). Four sets of hyper-chaotic sequences are obtained: $Q_{1}=\left\{q_{11}, q_{12}, \cdots, q_{1 \mathrm{~s}}\right\}, Q_{2}=\left\{q_{21}, q_{22}, \cdots, q_{2 \mathrm{~s}}\right\}, Q_{3}=\left\{q_{31}\right.$, $\left.q_{32}, \cdots, q_{3 \mathrm{~s}}\right\}$, and $Q_{4}=\left\{q_{41}, q_{42}, \cdots, q_{4 \mathrm{~s}}\right\}$. Each element in the hyper-chaotic sequence is pre-processed by formula (6), and the sequences $Q_{1}{ }^{\prime}, Q_{2}{ }^{\prime}, Q_{3}{ }^{\prime}$, and $Q_{4}{ }^{\prime}$ are obtained.

$$
f(x)=\bmod (x \times 1000,256)
$$

Step 4 Followed by the elements in sequences $Q_{1}{ }^{\prime}, Q_{2}{ }^{\prime}, Q_{3}{ }^{\prime}$, and $Q_{4}{ }^{\prime}$ to judge whether it falls into a sub-interval of $T$, assuming that the value of an element falls into the sub-interval $[j, j+1)$ and $j$ isn't in the $M_{1}$, then $j$ is appended to the $M_{1}$ until the number of elements in the $M_{1}$ reaches 256.

Step 5 Continue to judge the remaining elements in the sequences $Q_{1}{ }^{\prime}, Q_{2}{ }^{\prime}, Q_{3}{ }^{\prime}$, and $Q_{4}{ }^{\prime}$, and fill the sequence $M_{2}, \cdots$, $M_{L / 16}$. Ensure that each sequence contains 256 elements.

The $M$ sequence constructed in this way has randomness and can effectively resist plaintext attacks.

\subsection{Elliptic Curve}

Elliptic curve cryptography (ECC), an algorithm for establishing public key encryption. The main advantage of ECC is that in some cases it uses smaller keys, such as RSA encryption algorithms, to provide equivalent or higher security levels than other methods. Another advantage of ECC is that it is possible to define bilinear mappings between groups, based on Weil pairs or Tate pairs; bilinear mappings have found a large number of applications in cryptography, such as identity-based encryption. The disadvantage is that the implementation of encryption and decryption operations is more time-consuming than other mechanisms. In 2005, the NSA announced its decision to adopt a strategy of elliptic curve ciphers as part of the U.S. government's standards to protect sensitive information. Many forms of ECC are slightly different, and all depend on the widely recognized difficulty in solving discrete logarithm problems, corresponding to the group of elliptic curves on finite fields.

The elliptic curve refers to the plane curve determined by the Weierstrass equation:

$$
y_{2}+a_{1} x y+a_{3} y=x_{3}+a_{2} x_{2}+a_{4} x+a_{6}
$$


If $F$ is a domain, where $a_{i} \in F(i=1,2,3,4,6)$, the number $(x, y)$ that satisfies the Weierstrass equation is called the point of the elliptic curve in the $F$ domain. The $F$ domain is a rational domain or a complex domain. All points of the elliptic curve, plus a special infinite point $O$, are defined as the elliptic curve on the finite domain $F$.

In the applications of cryptography, there are two kinds of elliptic curves often used: the elliptic curves with prime values on the prime number domain $F_{p}$, where $P$ is an odd prime number, and the elliptic curve on the finite field $F_{2}{ }^{m}$ with an eigenvalue of 2 . The elliptic curve used in this paper is the eigenvalue with prime number domain $F_{p}$. In $F_{p}$, an elliptic curve $E$ with a large prime number factor is randomly searched, and $G_{0}=\left(x_{0}^{\prime}, y_{0}^{\prime}\right)$ is the base point of the prime number $p$ on $E$. $a$ and $b$ are the parameters of the elliptic curve $E$. The elliptic curve $E$ defined on the prime number domain $F_{p}$ is:

$$
y^{2}=x^{3}+a x+b(\bmod p)
$$

Where a and $\mathrm{b}$ satisfy $4 a^{2}+27 b^{3} \neq 0(\bmod p)$, and $p$ is the order of the elliptic curve. Then, the point that satisfies this equation and an infinity point $O$ forms an elliptic curve [23-24].

Two randomly selected numbers from the $M$ sequence are parameters $a$ and $b$ of the elliptic curve. If the selected $a=180, b=226, p=251$, then $E: y^{2}=x^{3}+180 x+226(\bmod 251)$, and $4 \times(180)^{2}+27 \times(226)^{3}=140 \neq 0(\bmod 251)$. The base point $G_{0}=(1,77)$ of the elliptic curve is obtained. The system parameter is $\left(F_{251},(1,77), 251,180,226\right)$.

Key generation: each user generates their own key, and the sender Alin randomly selects an integer $d_{a}$ as the private key in the $M$ sequence and $1 \leq d_{a} \leq p-1$. The receiver Bill randomly selects an integer $d_{b}$ as the private key in the $M$ sequence and $1 \leq d_{b} \leq p-1$.

The public key of both sides: if the sender Alin, $P_{a}=d_{a} G_{0}$, and the receiver Bill, $P_{b}=d_{b} G_{0}$, then $d_{a} \times P_{b}=d_{b} \times P_{a}=\left(x^{\prime}, y^{\prime}\right)$. It can be seen from the above algorithm that to obtain $\left(x^{\prime}, y^{\prime}\right)$, the $d_{a}$ of Alin and the $P_{b}$ of Bill must be obtained, or the private key $d_{b}$ of Bill and the public key $P_{a}$ of Alin must be obtained. According to the $G_{0}, P_{a}$, and $P_{b}$, the private key $d_{a}$ of Alin or the private key $d_{b}$ of user Bill is calculated. This is equivalent to solving the discrete logarithm problem of elliptic curves. As such, it is difficult to obtain $\left(x^{\prime}, y^{\prime}\right)$.

\subsection{The Construction of Hill Matrix}

The Hill cipher is a replacement cipher. To avoid the complexity of the elliptic curve, symmetric cryptography cannot effectively carry out key management, and the correlation between the encryption matrix elements is strong, leading to the easy cracking of the encryption system. In this paper, the elliptic curve and $M$ sequence are used to construct the encryption matrix.

By constructing an 8 by 8 reversible matrix $K$, the Hill cipher is conducted for each block of images. The specific equation is shown below:

$$
E=(K \times I) \bmod 256=\left[\begin{array}{ccc}
k_{11} & \cdots & k_{18} \\
\vdots & \ddots & \vdots \\
k_{81} & \cdots & k_{88}
\end{array}\right] \times\left[\begin{array}{c}
I_{11} \\
\vdots \\
I_{81}
\end{array}\right] \bmod 256=\left[\begin{array}{c}
E_{11} \\
\vdots \\
E_{81}
\end{array}\right]
$$

Where $E$ is the result of the Hill encryption, $I_{11 \sim 81}$ are a set of pixels to be encrypted, and $k_{11 \sim 88}$ are the Hill encryption matrix $K$; the ciphertext is decrypted by using the inverse matrix $K^{-1}$ of $K, I=\left(K^{-1} \times E\right) \bmod 256=(K \times E) \bmod 256$.

To avoid the complexity of the algorithm, the $G_{0}$ point and the $M$ sequence of the elliptic curve are combined to construct the self-inverse matrix $K$. The encryption matrix $K$ is divided into four parts:

$$
K=\left[\begin{array}{ll}
K_{11} & K_{12} \\
K_{21} & K_{22}
\end{array}\right], K_{11}=\left[\begin{array}{llll}
k_{11} & k_{12} & k_{13} & k_{14} \\
k_{21} & k_{22} & k_{23} & k_{24} \\
k_{31} & k_{32} & k_{33} & k_{34} \\
k_{41} & k_{42} & k_{43} & k_{44}
\end{array}\right]
$$

(1) According to the parameters of elliptic curve system, calculate $\left(x^{\prime}, y^{\prime}\right)=d_{a} \times P_{b}=d_{b} \times P_{a},\left(k_{11}, k_{12}\right)=x^{\prime} G_{0} ;\left(k_{13}\right.$, $\left.k_{14}\right)=m_{1} G_{0} ;\left(k_{21}, k_{22}\right)=m_{2} G_{0} ;\left(k_{23}, k_{24}\right)=m_{3} G_{0} ;\left(k_{31}, k_{32}\right)=m_{4} G_{0} ;\left(k_{33}, k_{34}\right)=m_{5} G_{0} ;\left(k_{41}, k_{42}\right)=m_{6} G_{0} ;\left(k_{43}, k_{44}\right)=y^{\prime} G_{0}$, and the submatrix $K_{11}$ is obtained. Among them, $m_{1}, m_{2}, \cdots, m_{6}$ are the first six elements in the $M_{1}$ sequence. 
(2) The sub-matrix $K_{11}$ is used as the following calculation to generate the sub-matrix $K_{12}, K_{12}=n \times\left(I-K_{11}\right)$.

(3) $K_{22}=-K_{11}$.

(4) $K_{21}=1 / \mathrm{n} \times\left(I+K_{11}\right)$. Then, the produced four sub-matrices $K_{11}, K_{12}, K_{22}$, and $K_{21}$ are combined to acquire the cipher matrix $K$.

The elements in the $M$ sequences are blocked every 16 times, and the $4 \times 4$ matrices are converted. The $4 \times 4$ matrices and $K_{11}$ are multiplied to form the first part of the encryption matrices. Repeat steps (2)-(4) to obtain encryption matrices $K_{1}$, $K_{2}, \cdots, K_{\mathrm{L}}$.

\subsection{Encryption Algorithm}

The Hill encryption matrix is constructed by combining an elliptic curve with a hyper-chaotic sequence; the image is ciphered, and the value of the plaintext is further diffused using dynamic DNA encoding technology. The specific flowchart is demonstrated in Figure 1 and the encryption processes are as shown below:

(1) Input the grayscale image matrix $I_{m n}$.

(2) Set each 8 pixels in a plaintext image is a block (if the last block is not enough 8 pixels, then fill 0). For each set of pixels, a matrix is selected from the Hill encryption matrix constructed in section 3.4 , and the matrix is permuted and encrypted to obtain the image matrix $I_{1}$.

(3) According to the formula (2), the DNA encoding rule is dynamically selected to convert the image matrix $I_{1}$ into the DNA sequence matrix $I_{2}$. Take the first column of the encryption matrix $K_{1}, K_{2}, \cdots, K_{\mathrm{L}}$, and synthesize a new matrix $K_{\mathrm{L}+1}$. According to formula (3), the corresponding coding rule is selected, and the matrix $K_{\mathrm{L}+1}^{\prime}$ is obtained by DNA encoding of the $K_{\mathrm{L}+1}$. The matrix $I_{3}$ is acquired by performing the DNA sequence operation on the matrix $I_{2}$ and the matrix $K_{\mathrm{L}+1}^{\prime}$.

(4) According to formula (3), DNA decoding of DNA sequence matrix $I_{3}$ is carried out, and the image matrix $I_{4}$ is obtained.

(5) Each gray value of the image matrix $I_{4}$ is represented in binary, and each pixel value in $I_{4}$ is cyclically shifted by 2 bits to obtain an image matrix $I_{5}$.

(6) The hyper-chaotic sequence $Q_{4}$ is extracted, and a novel sequence is arranged on the basis of the order from small to large. The element in the original sequence is permuted by the position of the elements in the new sequence, and the index sequence is obtained. The image matrix $I_{5}$ is scrambled by the index sequence, and the final encrypted image matrix $I_{6}$ is acquired.

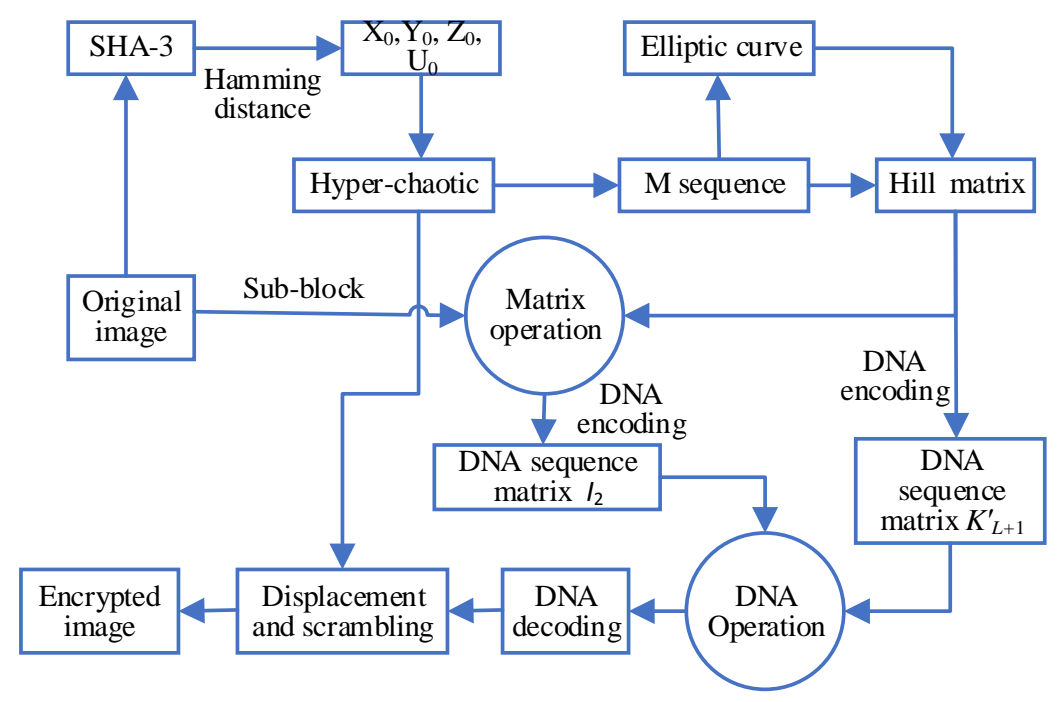

Figure 1. Encryption flowchart 
Decryption is the inverse procedure of encryption because the invertible matrix $K$ is a self-inverse matrix; therefore, the decryption algorithm does not need to calculate the inverse matrix $K$, which greatly saves computational time and computational complexity.

\section{Experimental Results and Security Analysis}

The experiments are conducted using the MATLAB 7.1(R2014a). The classical 256 by 256 Lena is utilized as the plaintext. By calculation, $x_{1}=63, y_{1}=25, z_{1}=47$ and $u_{1}=20.75$. The plaintext image and the ciphered image are demonstrated in Figure 2 . As shown in Figure 2(b), the original image is completely unrecognizable after being encrypted, indicating the validity of the encryption. As seen from the decryption result, the image can be restored without distortion, indicating the feasibility of the algorithm.

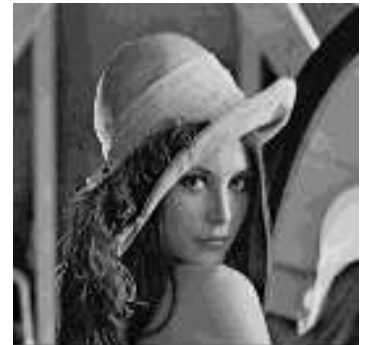

(a) Lena image

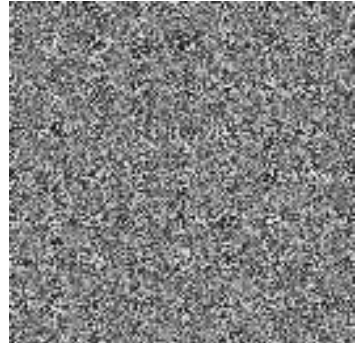

(b) Encrypted image

Figure 2. (a) Lena image; (b) Encrypted image

\subsection{Exhaustive Attack Analysis}

\subsubsection{Key Space Analysis}

In this encryption algorithm, the main keys include $x_{1}, y_{1}, z_{1}, u_{1}, a, b$, and the receiver key $d_{b}$. The key space is $10^{84} \times 2^{128} \approx 3.4 \times 10^{122}$, which indicates the method has a sufficient key space to defend exhaustive attack.

\subsubsection{Key Sensitivity Analysis}

The known key is modified slightly to verify the sensitivity of the image. Figure 3(a) represents the decryption diagram of $x_{1}=63.1$ with another key invariant. 3(b), 3(c), and 3(d) represent the decryption images of $y_{1}=25.1, z_{1}=47.1$, and $u_{1}=21$ with another key unchanged, respectively. Only if the decryption key is in line with the encryption key can the image be deciphered correctly. Otherwise, if there is a small change in the key, the plain image cannot be restored properly, and the error-deciphered image cannot mirror the plaintext image. As a result, the method has key sensitivity.

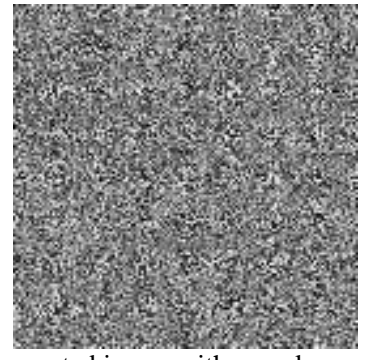

(a) Decrypted image with error key $x_{1}=63.1$

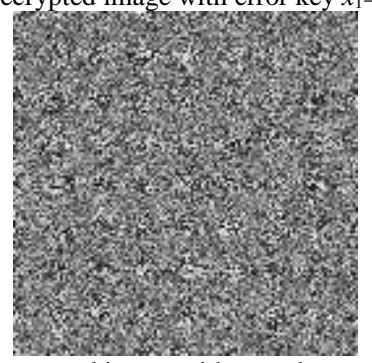

(b) Decrypted image with error key $z_{1}=47.1$

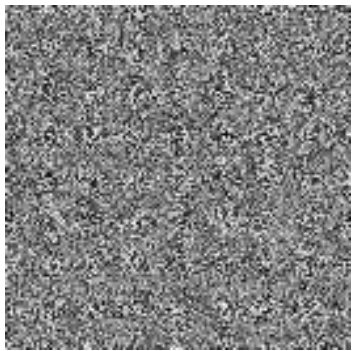

(c) Decrypted image with error key $y_{1}=25.1$

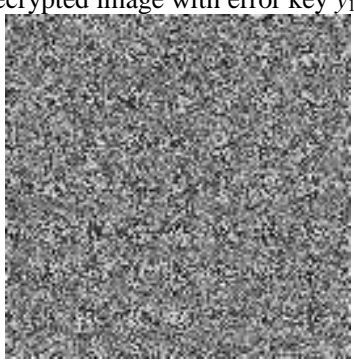

(d) Decrypted image with error key $u_{1}=21$

Figure 3. Decrypted image under the error key 


\subsection{Statistical Attack Analysis}

\subsubsection{Histogram Analysis}

A histogram is adopted to represent the distribution of gray values in grayscale images. Figure 4(a) represents the plain image histogram, and Figure 4(b) represents the histogram of the encrypted image. The pixels are relatively scattered before encryption, but after encryption, the corresponding histogram is almost unified; this shows that the algorithm can effectively resist statistical attack.

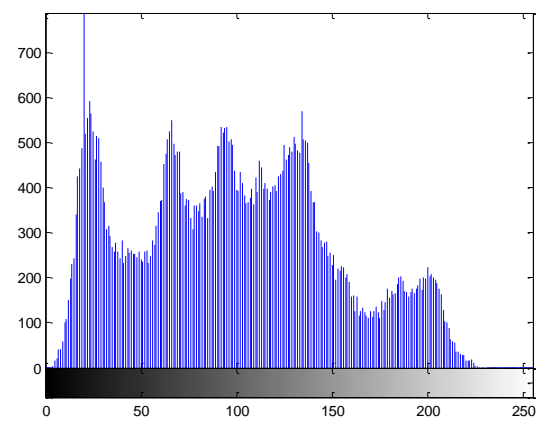

(a) Histogram of the Lena

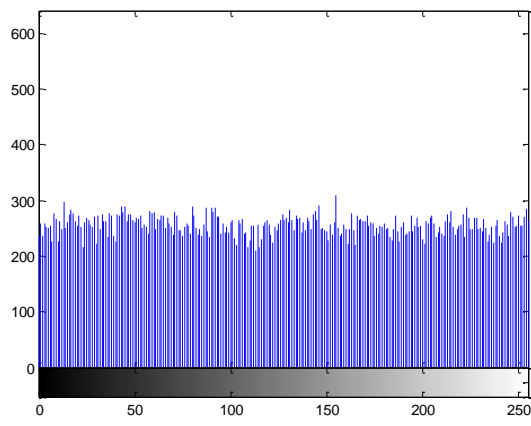

(b) Histogram of the ciphered Lena Figure 4. (a) Histogram of the Lena; (b) Histogram of the ciphered Lena

\subsubsection{Correlation Analysis}

To analyse the correlation coefficient between the pixels in the plain image and the ciphered image. 2500 pairs of adjacent pixels in three directions are randomly selected from the plain image and the ciphered image, and then formulas (11)-(14) are adopted to calculate the coefficient between pixels.

$$
\begin{gathered}
E(x)=\frac{1}{N} \sum_{i=1}^{N} x_{i} \\
D(x)=\frac{1}{N} \sum_{i=1}^{N}\left(x_{i}-E(x)\right)^{2} \\
\operatorname{COV}(x, y)=\frac{1}{N} \sum_{i=1}^{N}\left(x_{i}-E(x)\right)\left(y_{i}-E(y)\right) \\
\rho_{x y}=\frac{\operatorname{COV}(x, y)}{\sqrt{D(x)} \times \sqrt{D(y)}}
\end{gathered}
$$

Where $x$ and $y$ denote the values of the two adjacent pixels in the image, $E(x)$ is the average, $D(x)$ is the variance, and $\operatorname{COV}(x, y)$ is the covariance. By calculation, the correlation coefficient of this method is -0.0002342 . Table 3 shows the correlation coefficients in different directions. It can be seen from Figure 5 that the component of the original image has a great correlation in each direction, but after encrypting, the correlation of different components of the ciphertext image is very small. This can preserve the image information from being leaked; therefore, the image encryption algorithm can effectively defend against statistical attacks.

Table 3. Correlation coefficient of the plaintext image and the ciphered image

\begin{tabular}{|c|c|c|c|}
\hline Correlation coefficient & Horizontal & Vertical & Diagonal \\
\hline Plaintext & 0.9689 & 0.9486 & 0.9228 \\
\hline Ciphertext & -0.0029 & -0.0031 & -0.0037 \\
\hline Ref.[6] & -0.0045 & $-1.62 \mathrm{e}-04$ & 0.0053 \\
\hline Ref.[2] & 0.0042 & 0.0034 & 0.0016 \\
\hline Ref.[7] & 0.0041 & 0.0029 & 0.0019 \\
\hline
\end{tabular}




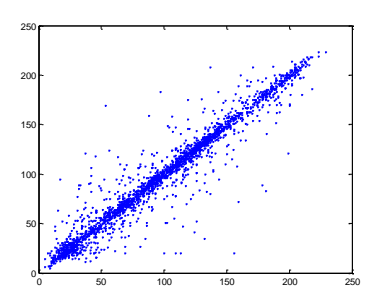

(a)

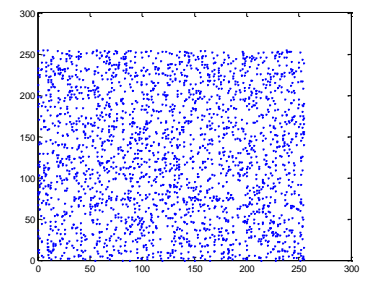

(d)

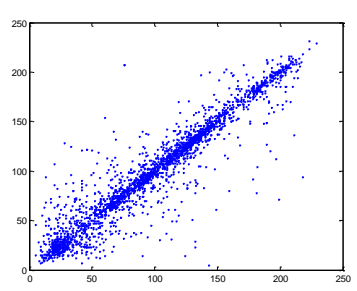

(b)

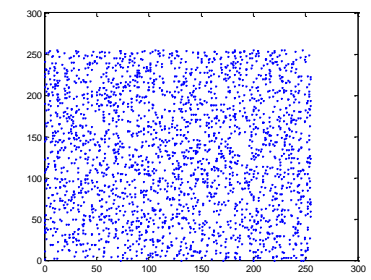

(e)

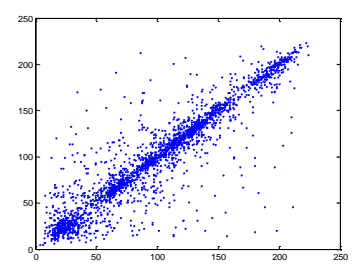

(c)

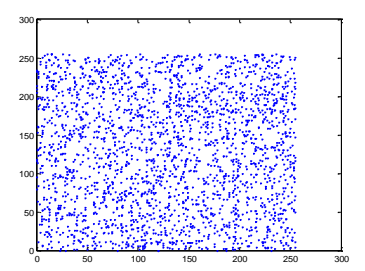

(f)

Figure 5. Correlation analysis: (a)-(c) Correlations of the plaintext image in three directions; (d)-(f) Correlations of the ciphered image in three directions

\subsubsection{Information Entropy Analysis}

Information entropy is a criterion to judge the uncertainty of a grayscale image. The specific calculation formula is shown below:

$$
H(m)=-\sum_{i=0}^{L} P\left(m_{i}\right) \log _{2} P\left(m_{i}\right), \sum_{i=0}^{L} P\left(m_{i}\right)=1
$$

Here, $P\left(m_{i}\right)$ denotes the probability that the information $m_{i}$ occurs. When the entropy approaches 8 , the produced images are completely random. The information entropy of this method is 7.9895 , as shown in Table 4 , indicating that the leakage probability of the ciphertext is very small and further verifies the security of the method.

Table 4. Comparison of information entropy in this paper and other references
\begin{tabular}{|c|c|c|c|}
\hline Information entropy & Lean & Ref.[28] & Ref.[15] \\
\hline H & 7.9895 & 7.9871 & 7.9874 \\
\hline
\end{tabular}

\subsection{Differential Attack Analysis}

The two criterions of number of pixels change rate (NPCR) and unified average changing intensity (UACI) are applied to weight whether the encryption technology can resist the differential attack. If the algorithm has a strong sensitivity to the plaintext, the higher the NPCR value, the closer the NPCR is to $100 \%$, showing that the method is more likely to defend against differential attacks. UACI represents the ratio of the average change of the pixel values of all the plain images and the corresponding positions of the ciphertext images. The closer the UACI value is to $33 \%$, the stronger the ability to defend against differential attacks. The equations below are utilized to compute NPCR and UACI:

$$
\begin{gathered}
C(i, j)= \begin{cases}0, & \text { if } P_{1}(i, j)=P_{2}(i, j) \\
1, & \text { if } P_{1}(i, j) \neq P_{2}(i, j)\end{cases} \\
N P C R=\frac{\sum_{i=1}^{M} \sum_{j=1}^{N} C(i, j)}{M \times N} \times 100 \% \\
U A C I=\frac{\sum_{i=1}^{M} \sum_{j=1}^{N}\left|P_{1}(i, j)-P_{2}(i, j)\right|}{255 \times M \times N} \times 100 \%
\end{gathered}
$$

Where $M$ and $N$ denote the length and width of the image, respectively. $P_{1}(i, j)$ represents the pixel values of the plaintext image, and $P_{2}(i, j)$ signifies the pixel values of the ciphertext image. For the Lena images shown in Table 5, the NPCR and UACI of the algorithm are $99.6 \%$ and $33.38 \%$, respectively, demonstrating that the encryption method has the capacity to defend against differential attacks. 
Table 5. Comparison of NPCR and UACI in this paper and other references

\begin{tabular}{|c|c|c|}
\hline & NPCR & UACI \\
\hline Ours & $99.6 \%$ & $33.38 \%$ \\
\hline Ref.[8] & $99.0276 \%$ & $32.8399 \%$ \\
\hline Ref.[6] & $99.59 \%$ & $33.41 \%$ \\
\hline Ref.[7] & $99.1390 \%$ & $32.2306 \%$ \\
\hline Ref.[15] & $99.60 \%$ & $28.13 \%$ \\
\hline
\end{tabular}

\section{Conclusions}

The Hill encryption matrix is constructed by the combination of an elliptic curve and a hyper-chaotic system, thereby avoiding the complexity of elliptic curve encryption and the correlation between encryption matrix elements. This simplifies the encryption method and allows for randomness in the encryption matrix. Combined with dynamic DNA encoding rules, the security of the encryption method is increased and the correlation between pixels is reduced, making the ciphertext difficult to crack. The simulation and experimental results indicate that this algorithm has a better encryption result, larger key space, and higher sensitivity to the key. Furthermore, the algorithm can defend against exhaustive attacks, statistical attacks, and differential attacks.

\section{Acknowledgements}

The work for this paper was supported by the National Natural Science Foundation of China (Grant Nos. 61472371, 61572446, 61602424, and 61472372), the Program for Science and Technology Innovation Talents in Universities of Henan Province (Grant No. 15HASTIT019), the Key Scientific Research Projects of Henan High Educational Institution (Grant No. 18A510020), and the Plan for Scientific Innovation Talent of Henan Province (Grant No. 174100510009).

\section{References}

1. X. Chai, Y. Chen, and L. Broyde, "A Novel Chaos-based Image Encryption Algorithm using DNA Sequence Operations," Optics \& Lasers in Engineering, Vol. 88, pp. 197-213, 2017

2. R. Enayatifar, H. J. Sadaei, A. H. Abdullah, M. Lee, and I. F. Isnin, "A Novel Chaotic based Image Encryption using a Hybrid Model of Deoxyribonucleic Acid and Cellular Automata," Optics \& Lasers in Engineering, Vol. 71, pp. 33-41, 2015

3. X. Wang, J. Zhao, and H. Liu, "A New Image Encryption Algorithm based on Chaos," Optics Communications, Vol. 285, No. 5, pp. 562-566, 2012

4. F. Özkaynak and S. Yavuz, "Analysis and Improvement of a Novel Image Fusion Encryption Algorithm based on DNA Sequence Operation and Hyper-chaotic System," Nonlinear Dynamics, Vol. 78, No. 2, pp. 1311-1320, 2014

5. L. Kong and L. Li, "A New Image Encryption Algorithm based on Chaos," in Proceedings of 35th Chinese Control Conference (CCC), pp. 4932-4937, 2016

6. F. Özkaynak, A. B. Özer, and S. Yavuz, "Security Analysis of an Image Encryption Algorithm based on Chaos and DNA Encoding," in Proceedings of Signal Processing and Communications Applications Conference (SIU), pp. 1-4, 2013

7. B. Santhosh and K. Viswanath, "A Novel Public Key Cryptosystem for Medical Images," in Proceedings of International Conference on Inventive Systems and Control (ICISC), pp. 1-4, 2017

8. K. Gupta, S. Silakari, R. Gupta, and S. A. Khan, "An Ethical Way of Image Encryption using ECC," in Proceedings of First International Conference on Computational Intelligence, Communication Systems and Networks (CICSYN), pp. 342-345, 2009

9. A. Soleymani, J. Nordin, A. N. Hoshyar, Z. M. Ali, and E. Sundararajan, "An Image Encryption Scheme based on Elliptic Curve and a Novel Mapping Method," International Journal of Digital Content Technology \& its Applications, Vol. 7, No. 13, pp. 85, 2013

10. F. Wang, X. Q. Zhang, and G. L. Zhu, "A Block Image Element Encryption Algorithm based on ECC," Communications Technology, Vol. 41, No. 3, pp. 82-84, 2008

11. L. J. Chen and A. D. Shen, "A Novel Public Key Image Cryptosystem based on Elliptic Curve and Arnold Cat Map," Advanced Materials Research, Vol. 989-994, pp. 4183-4186, 2014

12. M. Kumar, A. Iqbal, and P. Kumar, "A New RGB Image Encryption Algorithm based on DNA Encoding and Elliptic Curve Diffie-hellman Cryptography,” Signal Processing, Vol. 125, No. C, pp. 187-202, 2016

13. B. Acharya, S. K. Patra, and G. Panda, "Image Encryption by Novel Cryptosystem using Matrix Transformation," in Proceedings of International Conference on Emerging Trends in Engineering and Technology, pp. 77-81, 2008

14. R. Mahendran and K. Mani, "Generation of Key Matrix for Hill Cipher Encryption using Classical Cipher," in Proceedings of World Congress on Computing and Communication Technologies (WCCCT), pp. 51-54, 2017

15. K. D. Patel and S. Belani, "Image Encryption using Different Techniques," International Journal of Emerging Technology and Advanced Engineering, Vol. 1, No. 1, pp. 30-34, 2011

16. A. A. A. Gutub and F. A. A. Khan, "Hybrid Crypto Hardware Utilizing Symmetric-Key and Public-Key Cryptosystems," in Proceedings of International Conference on Advanced Computer Science Applications and Technologies, pp. 116-121, 2013

17. X. Wang and M. Wang, "A Hyperchaos Generated from Lorenz System," Physica A Statistical Mechanics \& its Applications, Vol. 387, No. 14, pp. 3751-3758, 2008 
18. R. Enayatifar, A. H. Abdullah, and I. F. Isnin, "Chaos-based Image Encryption using a Hybrid Genetic Algorithm and a DNA Sequence," Optics \& Lasers in Engineering, Vol. 56, No. 5, pp. 83-93, 2014

19. H. Liu, X. Wang, and A. Kadir, "Image Encryption using DNA Complementary Rule and Chaotic Maps," Applied Soft Computing Journal, Vol. 12, No. 5, pp. 1457-1466, 2012

20. I. I. Cisse, H. Kim, and T. Ha, "A Rule of Seven in Watson-Crick base Pairing of Mismatched Sequences," Nature Structural \& Molecular Biology, Vol. 19, No. 6, pp. 623-627, 2012

21. R. Guesmi, M. A. B. Farah, A. Kachouri, and M. Samet, "A Novel Chaos-based Image Encryption using DNA Sequence Operation and Secure Hash Algorithm SHA-2," Nonlinear Dynamics, Vol. 83, No. 3, pp. 1-14, 2015

22. G. Cui, Y. Liu, X. Zhang, and Z. Zhou, "A New Image Encryption Algorithm based on DNA Dynamic Encoding and HyperChaotic System," in Proceedings of International Conference on Bio-Inspired Computing: Theories and Applications, pp. 286-303, 2017

23. B. K. Alese, E. D. Philemon, and S. O. Falaki, "Comparative Analysis of Public-Key Encryption Schemes," International Journal of Engineering \& Technology, Vol. 2, No. 9, pp. 1552-1568, 2012

24. J. Hoffstein, J. Pipher, and J. H. Silverman, "An Introduction to Mathematical Cryptography," 2nd Edition, Springer Publishing Company, 2014 